\title{
Detection of epidermal growth factor receptor mutations in circulating tumor DNA: reviewing BENEFIT clinical trial
}

\author{
Umberto Malapelle ${ }^{1}$, Luis E. Raez ${ }^{2}$, Maria Jose Serrano ${ }^{3,4}$, Christian Rolfo ${ }^{5}$ International Society of \\ Liquid Biopsies (ISLB)
}

\begin{abstract}
${ }^{1}$ Department of Public Health, University Federico II of Naples, Italy; ${ }^{2}$ Memorial Cancer Institute/Memorial Health Care System Florida International University, Miami, Florida, USA; ${ }^{3}$ Integral Oncology Division, Clinical University Hospital, Granada, Spain; ${ }^{4}$ Liquid biopsy and metastasis research group, GENYO, Centre for Genomics and Oncological Research, Pfizer/University of Granada/Andalusian Regional Government PTS, Granada, Spain; ${ }^{5}$ Marlene and Stewart Greenebaum Comprehensive Cancer Center, University of Maryland School of Medicine, Baltimore, Maryland, USA

Correspondence to: Prof. Dr. Christian Rolfo, MD, PhD, MBA, hc. University of Maryland Marlene and Stewart Greenebaum Comprehensive Cancer Center, University of Maryland School of Medicine, Baltimore, Maryland, USA. Email: christian.rolfo@umm.edu.

Provenance: This is an invited Editorial commissioned by the Section Editor Dr. Lei Deng (Department of Medicine, Jacobi Medical Center, Albert Einstein College of Medicine, Bronx, NY, USA).

Comment on: Wang Z, Cheng Y, An T, et al. Detection of EGFR mutations in plasma circulating tumour DNA as a selection criterion for first-line gefitinib treatment in patients with advanced lung adenocarcinoma (BENEFIT): a phase 2, single-arm, multicentre clinical trial. Lancet Respir Med 2018;6:681-90.
\end{abstract}

Submitted Oct 29, 2018. Accepted for publication Nov 06, 2018.

doi: $10.21037 /$ jtd.2018.11.22

View this article at: http://dx.doi.org/10.21037/jtd.2018.11.22

The identification of epidermal growth factor receptor (EGFR) sensitizing mutations plays a key role in the management of non-small cell lung cancer (NSCLC) patients (1-4). Tissue specimens are not always available, due to difficulty in obtaining enough material, despite the fact that more than $70 \%$ of NSCLC patients are diagnosed in the metastatic stage $(5,6)$ due to multiple reasons. In this setting, prior to the administration of any biological or chemotherapy treatment regimen, liquid biopsy could represent a valid alternative to tissue specimens, taking in consideration the need to use appropriate molecular techniques. In addition, after treatment with first and/or second-generation tyrosine kinase inhibitors (TKIs), the arising of EGFR resistance mutations (in particular the $E G F R$ exon 20 p.T790M) allows the treatment with third generation TKI (e.g., osimertinib) (7). Moreover, the collection of new tissue specimens after a first line treatment to detect EGFR resistance mutations and other druggable genetic alteration is challenging and time consuming. For these reasons, the European Medicines Agency (EMA) and Food and Drug Administration (FDA) approved the analysis of EGFR status on circulating tumor DNA (ctDNA) extracted from plasma samples in new NSCLC patients without tissue availability or after resistance to first and/or generation TKIs for the detection of EGFR exon 20 p.T790M $(8,9)$.

The main challenge is represented by the low frequency of ctDNA, passively or actively released by tumor, into the bloodstream $(<0.5 \%)$ respect to the total released cell free DNA (cfDNA) $(6,10,11)$. Careful attention was paid on the technologies to adopt in order to avoid false positive (high specificity) and/or false negative (high sensitivity) results (6). In most of the clinical trials the principal methodology adopted was real-time polymerase chain reaction (RT-PCR) and the digital droplet PCR (ddPCR) (9-11). More recently, in other experiences, a next generation sequencing (NGS) approach was evaluated to achieve better results in term of sensitivity and specificity by using as a gold standard the EGFR mutational status evaluated on tissue samples (9-13).

Recently, in Lancet Respiratory Medicine journal, Wang et al. showed the results of an open-label, single arm, prospective, multicentre, phase 2 clinical trial (BENEFIT) with the aim to assess the feasibility of EGFR detection, by ddPCR, in ctDNA extracted from pre-treated NSCLC adenocarcinoma (ADC) patients' plasma, for the administration of gefitinib as first-line treatment (14). From December 25, 2014 to January 16, 2016, 188 out 


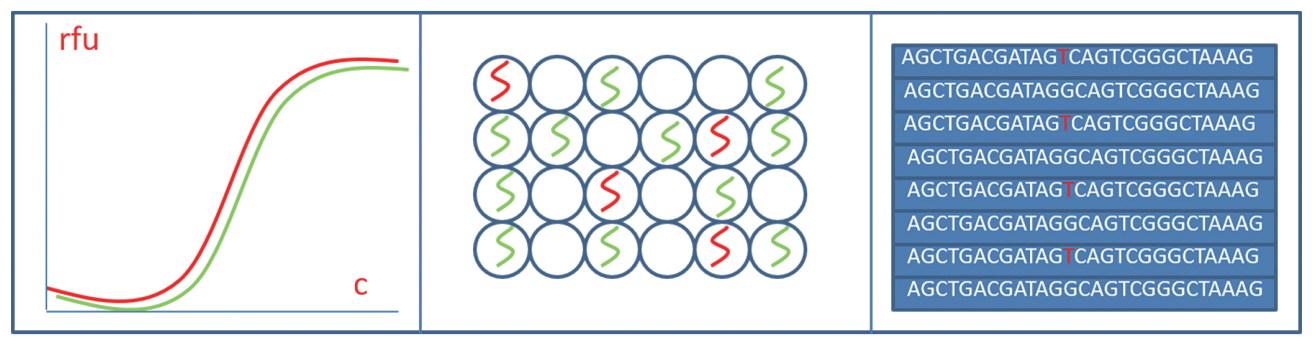

Figure 1 Graphical representation of main molecular biology techniques used in the reported studies. In the left panel was represented wildtype (green) and mutated (red) RT-PCR amplification curves. In the center was reported an example of digital PCR compartmentalization with wild-type (green) and mutated (red) separated alleles. In the right panel was reported a schematization of NGS sequences (reads) pil-up for a visual inspection of mutated (read "T") alleles. rfu, relative fluorescence unit; c, cycles; RT-PCR, real-time polymerase chain reaction; NGS, next generation sequencing.

of 426 screened patients showed an EGFR sensitizing mutation and received gefitinib. In this study the primary end point was the objective response rate (ORR), and the secondary aim was the assessment of progression free survival (PFS), disease control, overall survival (OS) and safety and tolerability of gefitinib. Of these 188 patients, 180 (95.7\%) had the EGFR mutation both on ctDNA and tissue samples, the remaining $4.3 \%$ showed the mutation only on ctDNA. ORR was $72.1 \%$ (95\% CI: $65.0-78.5)$ and the median PFS was 9.5 months (95\% CI: 9.07-11.04). Other studies have analyzed prospectively the ctDNA but the EGFR mutational assessment on ctDNA was not adopted as an inclusion criterion $(12,15)$. Another study adopted a retrospective approach: Karachaliou et al. analyzed $\mathrm{N}=76$ blood samples with a multiplex 5 ' nuclease RT PCR (Taqman) assay to be used in the presence of a peptide nucleic acid (PNA) clamp (12). They showed, in particular, a negative prognostic value of the EGFR exon 21 p.L858R when detected on ctDNA respect to EGFR exon 19 deletions (12). In the experience of Mayo-de-LasCasas et al., 1,026 blood samples, without tissue specimens, of NSCLC patients at baseline were analyzed by a multiplex 5' nuclease real-time PCR (Taqman) assay to be used in the presence of a PNA clamp for wild-type (15). In this study the authors demonstrated that on a large-scale testing of an unselected population the clinical outcomes to TKIs in blood are undistinguishable from those obtained in tumour tissue. Also, Kimura et al. showed on 42 paired blood and tissue samples, by using Scorpion Amplification Refractory Mutation System (ARMS), that it is feasible to use DNA extracted from serum to assess EGFR mutational status and allow the treatment with TKIs (16). Similar to what was seen in these studies, Wang et al. concluded that the assessment of EGFR mutational status on ctDNA is suitable for the administration of TKIs. The main limitation of the discussed study is based on the use of molecular techniques with a limited multiplexing power: in fact, also if the modified RT PCR (such as PNART PCR) reaching a good level of sensitivity, showed a poor level reference range, not being able to identify all the possible clinically relevant gene alterations (13).

In a retrospective experience by Malapelle et al. showed that a narrow NGS panel $\left(\mathrm{SiRe}^{\circledR}\right)$ is a feasible tool for ctDNA analysis in clinical practice, and by adopting ctDNA extracted from both plasma and serum they obtained a sensitivity of $90.5 \%$ and a specificity of $100 \%$ (13). As shown by Wang et al. by using digital droplet PCR approach, also Pisapia $e t$ al. by using ultra-deep NGS, in a prospective series showed a key role of the analysis of ctDNA for EGFR molecular assessment and subsequently treatment decision for NSCLC patients in the basal setting (patients at diagnosis without tissue availability), but in addition to Wang et al., in the experience of Pisapia et al., in addition to the EGFR mutational status is also possible the simultaneous evaluation of other clinical relevant gene alteration in NSCLC patients, such as KRAS, NRAS and BRAF mutations (9). Raez et al. showed that in real life even doing NGS we not always get all the genetic aberrations in tissue (17). Comparing tissue with liquid biopsies (Guardant 360) in NSCLC patients, this group showed that we were able to get molecular markers in tissue in only $78 \%$ of the cases mainly due to insufficient tissue, and we needed liquid biopsies to complement the other $22 \%$ of the patients, moreover up to $1 / 3$ of all the actionable mutations were found only in plasma NGS but not in tissue NGS (17).

Beyond the role of specific molecular techniques (Figure 1), considering all together (Table 1) the data obtained from 


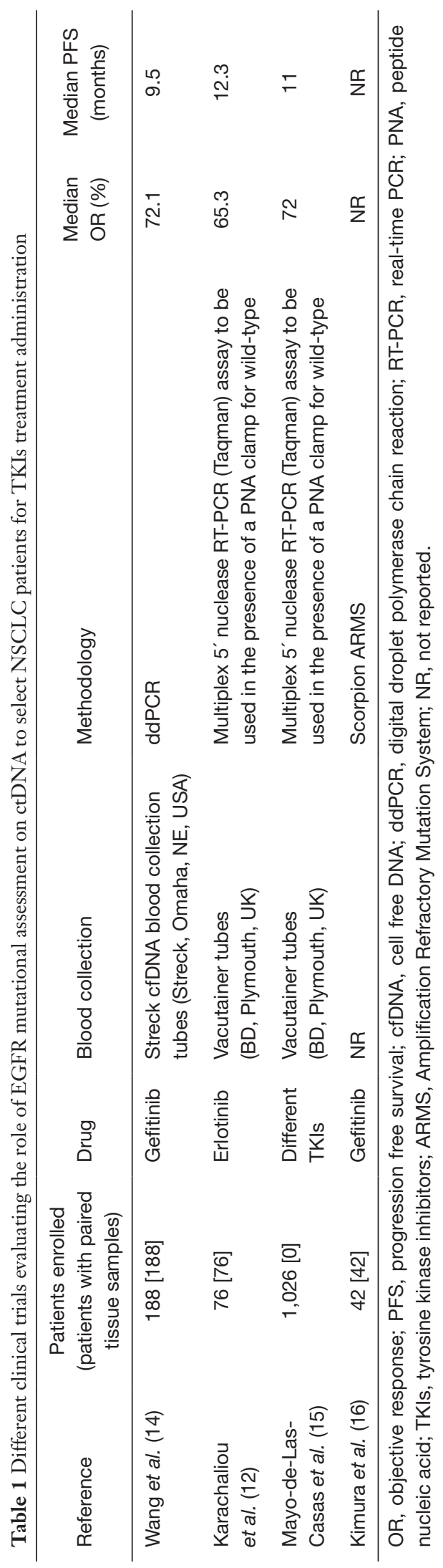

the experiences analyzed in this editorial and taking in to account the recent evidences obtained from the FLAURA clinical trial, a phase 3 trial that confirmed in new patients with a sensitizing EGFR mutation (ex19del or p.L858R), that osimertinib has a high efficacy respect to gefitinib or erlotinib as first-line treatment in advanced NSCLCs, the role of EGFR mutation assessment on ctDNA in basal setting became even more important in clinical setting respect to the role that today have to define the second line treatment after an EGFR TKI first generation administration (18-20).

In conclusion, as shown in the analyzed studies, the identification of $E G F R$ mutations in ctDNA plays a crucial role to better identify patients who might benefit from TKIs treatment option comparably with the data obtained starting from tissue extracted DNA. In particular, following the approval of osimertinib as first line treatment, the analysis of EGFR mutational status on ctDNA recovered from basal setting NSCLC patients may represent a key weapon in patient population without tissue specimens form molecular testing, taking into account the implementation of more sensitive techniques, such digital PCR and ultradeep NGS.

\section{Acknowledgements}

We acknowledge all the member of International Society of Liquid Biopsies (ISLB).

\section{Footnote}

Conflicts of Interest: The authors have no conflicts of interest to declare.

\section{References}

1. Lynch TJ, Bell DW, Sordella R, et al. Activating mutations in the epidermal growth factor receptor underlying responsiveness of non-small-cell lung cancer to gefitinib. N Engl J Med 2004;350:2129-39.

2. Mok TS, Wu YL, Thongprasert S, et al. Gefitinib or carboplatin-paclitaxel in pulmonary adenocarcinoma. $\mathrm{N}$ Engl J Med 2009;361:947-57.

3. Rosell R, Carcereny E, Gervais R, et al. Erlotinib versus standard chemotherapy as first-line treatment for European patients with advanced EGFR mutation-positive non-small-cell lung cancer (EURTAC): a multicentre, open-label, randomised phase 3 trial. Lancet Oncol 
2012;13:239-46.

4. Yang JC, Wu YL, Schuler M, et al. Afatinib versus cisplatin-based chemotherapy for EGFR mutation-positive lung adenocarcinoma (LUX-Lung 3 and LUX-Lung 6): analysis of overall survival data from two randomised, phase 3 trials. Lancet Oncol 2015;16:141-51.

5. Malapelle U, Bellevicine C, De Luca C, et al. EGFR mutations detected on cytology samples by a centralized laboratory reliably predict response to gefitinib in nonsmall cell lung carcinoma patients. Cancer Cytopathol 2013;121:552-60.

6. Malapelle U, Pisapia P, Rocco D, et al. Next generation sequencing techniques in liquid biopsy: focus on nonsmall cell lung cancer patients. Transl Lung Cancer Res 2016;5:505-10.

7. Jänne PA, Yang JC, Kim DW, et al. AZD9291 in EGFR inhibitor-resistant non-small-cell lung cancer. N Engl J Med 2015;372:1689-99.

8. Kwapisz D. The first liquid biopsy test approved. Is it a new era of mutation testing for non-small cell lung cancer? Ann Transl Med 2017;5:46.

9. Pisapia P, Pepe F, Smeraglio R, et al. Cell free DNA analysis by $\mathrm{SiRe} \AA$ next generation sequencing panel in non small cell lung cancer patients: focus on basal setting. J Thorac Dis 2017;9:S1383-90.

10. Schwarzenbach H, Hoon DS, Pantel K. Cell-free nucleic acids as biomarkers in cancer patients. Nat Rev Cancer 2011;11:426-37.

11. Crowley E, Di Nicolantonio F, Loupakis F, et al. Liquid biopsy: monitoring cancer-genetics in the blood. Nat Rev Clin Oncol 2013;10:472-84.

12. Karachaliou N, Mayo-de las Casas C, Queralt C, et al. Association of EGFR L858R Mutation in Circulating Free
DNA With Survival in the EURTAC Trial. JAMA Oncol 2015;1:149-57.

13. Malapelle U, Mayo de-Las-Casas C, Rocco D, et al. Development of a gene panel for next-generation sequencing of clinically relevant mutations in cell-free DNA from cancer patients. Br J Cancer 2017;116:802-10.

14. Wang Z, Cheng Y, An T, et al. Detection of EGFR mutations in plasma circulating tumour DNA as a selection criterion for first-line gefitinib treatment in patients with advanced lung adenocarcinoma (BENEFIT): a phase 2, single-arm, multicentre clinical trial. Lancet Respir Med 2018;6:681-90.

15. Mayo-de-Las-Casas C, Jordana-Ariza N, Garzón-Ibañez $M$, et al. Large scale, prospective screening of EGFR mutations in the blood of advanced NSCLC patients to guide treatment decisions. Ann Oncol 2017;28:2248-55.

16. Kimura H, Suminoe M, Kasahara K, et al. Evaluation of epidermal growth factor receptor mutation status in serum DNA as a predictor of response to gefitinib (IRESSA). Br J Cancer 2007;97:778-84.

17. Raez LE, Mekhail T, Rodriguez E, et al. L. MA 15.02 Plasma CfDNA next Generation Sequencing in NonSmall Cell Lung Cancer: Clinical Outcomes and Comparison to Tissue. J Thorac Oncol 2017;12:S1862.

18. Soria JC, Ohe Y, Vansteenkiste J, et al. Osimertinib in Untreated EGFR-Mutated Advanced Non-Small-Cell Lung Cancer. N Engl J Med 2018;378:113-25.

19. Malapelle U, Ricciuti B, Baglivo S, et al. Osimertinib. Recent Results Cancer Res 2018;211:257-76.

20. Rolfo C, Mack PC, Scagliotti GV, et al. Liquid Biopsy for Advanced Non-Small Cell Lung Cancer (NSCLC): A Statement Paper from the IASLC. J Thorac Oncol 2018;13:1248-68.
Cite this article as: Malapelle U, Raez LE, Serrano MJ, Rolfo C; International Society of Liquid Biopsies (ISLB). Detection of epidermal growth factor receptor mutations in circulating tumor DNA: reviewing BENEFIT clinical trial. J Thorac Dis 2018;10(12):6388-6391. doi: 10.21037/jtd.2018.11.22 